Для цитирования:

Мухтарова 3.М. Фазовые равновесия в системе $\mathrm{YbTe}-\mathrm{Yb}_{3} \mathrm{Ge}_{5}$. Изв. вузов. Химия и хим. технология. 2017. Т. 60.

Вып. 1. С. 64-67.

For citation:

Mukhtarova Z.M. Phase equilibrium in Ybte- $\mathrm{Yb}_{3} \mathrm{Ge}_{5}$ system. Izv. Vyssh. Uchebn. Zaved. Khim. Khim. Tekhnol. 2017. V. 60. N 1.P. 64-67.

УДК 546.668.289.24

\title{
3.M. Мухтарова
}

Зияфат Мамед кызы Мухтарова

Институт катализа и неорганической химии им. акад. М. Нагиева НАН Азербайджана, Баку, пр. Г. Джавида, 113, AZ1143

E-mail: iradam@rambler.ru

\section{ФАЗОВЫЕ РАВНОВЕСИЯ В СИСТЕМЕ YbТе - $\mathrm{Yb}_{3} \mathrm{Ge}_{5}$}

Методами физико-химического анализа ДТА, РФА, МСА, а также измерением микротвердости изучена система $\mathrm{YbTe}-\mathrm{Yb}_{3} G e_{5}$, которая является квазибинарным сечением тройной системы Ge-Te - Yb. Установлено, что диаграмма состояния системы является квазибинарной и относится к эвтектическому типу с монотектикой. Коорди-

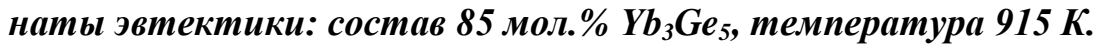

Ключевые слова: полупроводник, неквазибинарное, квазибинарное сечение, микротвердость, эвтектика, фазовая диаграмма

UDC: 546.668 .289 .24

\section{Z.M. Mukhtarova}

Ziyafat M. Mukhtarova

Institute of Catalysis and Inorganic Chemistry named after acad. M. Nagiyev, Azerbaijan National Academy of Sciences, Javid Ave., 113, Baku, Az1143, Azerbaijan

E-mail: iradam@rambler.ru

\section{PHASE EQUILIBRIUM IN YbTe-Y $\mathrm{Yb}_{3} \mathrm{Ge}_{5}$ SYSTEM}

Study of properties of semiconductors developed in close connection with their technical applications. The present work was devoted to study of phase equilibria and character of interaction in $\mathrm{YbTe}-\mathrm{Yb}_{3} \mathrm{Ge}_{5}$ system. The section of $\mathrm{YbTe}-\mathrm{Yb}_{3} G e_{5}$ in ternary system $\mathrm{Ge}-\mathrm{Te}-\mathrm{Yb}$ is not only scientific, but also practical interest. The section of $\mathrm{YbTe}-\mathrm{Yb}_{3} \mathrm{Ge}_{5}$ was studied by methods of physical-chemical analysis: differential-thermal (DTA), high temperature differential-thermal (HTDT), X-ray phase, microstructural analysis (MSA), as well as measurement of density and micro hardness. DTA was performed with pyrometer HTP-75 in quartz ampoule pimped off till $0.1333 \mathrm{~Pa}$. HTDT was performed with HTDT-8m (Tmelt. $\geq 1500 \div 2000 \mathrm{~K})$ by analogical method. $X$ ray phase analysis was performed by powder method with $X$-ray diffractometer DRON-2 (CuKaradiation with Ni-filter). MSA was performed with microscope MIM 8. Micro hardness of alloys 
was measured with micro hardness tester PMT-3.Density of alloys was determined by pycnometer test. During investigations of the system we used germanium $B-4$, tellurium $B-3$, ytterbium $Y b-1$. Alloys were synthesized at 1450-1700 K temperature range and at this temperature ampoule was kept 5-6 h. Cooling was performed slowly. DTA shows that on thermograms of alloys of the system have two effects. Obtained effects are endothermic reversible.For confirming the data of DTA, microstructural analysis, as well as measurement of micro hardness were performed with $X$-ray analysis. As the data show, at the concentration of 15-80 mol\% of $\mathrm{Yb}_{3} \mathrm{Ge}_{5}$ monotectic conversion occurs which is confirmed with isothermal line at $1025 \mathrm{~K}$. Thus, it was established that the section of $4 \mathrm{YbTe}-\mathrm{Yb}_{3} \mathrm{Ge}_{5}$ is quasibinary cross-section of ternary system Ge-Te-Yb and its diagram is related to eutectic type with monotectics. Eutectic of the system $4 \mathrm{YbTe}_{\mathrm{Y}} \mathrm{Yb}_{3} \mathrm{Ge} \mathrm{e}_{5}$ corresponds to composition of $85 \%$ mol\% of $\mathrm{Yb}_{3} \mathrm{Ge}_{5}$ and temperature of $915 \mathrm{~K}$.

Key words: semiconductor, non-quasi binary, quasi binary sections, microhardness, eutectic, phase diagram

В последние годы требования техники к полупроводниковым материалам значительно возросли. Учение о свойствах полупроводников развивалось в тесной связи с их техническими применениями $[1,2]$.

Большая роль в создании перспективных материалов отводится полупроводниковым системам с участием теллуридов редкоземельных элементов.

Однако узость интервала рабочих температур, плавления и механической прочности ограничивают возможности их практического применения.

Для достижения указанной цели работы необходимо изучение фазовых равновесий и характера взаимодействий в системе $\mathrm{YbTe}-\mathrm{Yb}_{3} \mathrm{Ge}_{5}$ с построением фазовой диаграммы, определением областей гомогенности и новых полупроводниковых фаз.

Разрез $\mathrm{YbTe}-\mathrm{Yb}_{3} \mathrm{Ge}_{5}$ в тройной системе $\mathrm{Ge}-\mathrm{Te}-\mathrm{Yb}$ представляет не только научный, но и практический интерес.

YbTe. Эта система известна образованием одного соединения YbTe. Соединение YbTe авторы $[3,4]$ получили из элементов ампульным методом. Температура плавления $\mathrm{YbTe}=2003$ К. ҮbTe кристаллизуется в кубической сингонии типа $\mathrm{NaCl}$, параметры решетки а $=6,37 \AA$ [5].

$\mathbf{Y b}_{3} \mathbf{G e}_{5}$. Соединение $\mathrm{Yb}_{3} \mathrm{Ge}_{5}$ плавится конгруэнтно при 1355 К и претерпевает полиморфное превращение $\alpha \rightleftarrows \beta$ при $1200 \mathrm{~K}$, существует в виде двух модификаций: $\alpha-\mathrm{Yb}_{3} \mathrm{Ge}_{5}$ и $\beta-\mathrm{Yb}_{3} \mathrm{Ge}_{5}$. Соединение $\alpha-\mathrm{Yb}_{3} \mathrm{Ge}_{5}$ имеет кристаллическую структуру типа $\mathrm{Th}_{3} \mathrm{~Pb}_{5}, \beta-\mathrm{Yb}_{3} \mathrm{Ge}_{5}$ относится к гексагональной сингонии с параметрами решетки $\mathrm{a}=6,847$; $\mathrm{c}=4,076 \AA$, пространственная группа (пр.гр.) $\mathrm{P} 62 \mathrm{~m}$. Сингония $\mathrm{Yb}_{3} \mathrm{Ge}_{5}$ гексагональная.

Для определения характера взаимодействия в тройной системе $\mathrm{Ge}-\mathrm{Te}-\mathrm{Yb}$ [6] были исследо- ваны четыре квазибинарных разреза $\mathrm{GeTe}-\mathrm{YbTe}$, $\mathrm{GeTe}-\mathrm{Yb}_{3} \mathrm{Ge}_{5}$ [7], $\mathrm{Yb}_{3} \mathrm{Ge}_{7} \mathrm{Te}_{2}-\mathrm{Ge}, \mathrm{Yb}_{3} \mathrm{Ge}_{7} \mathrm{Te}_{2}-$ $\mathrm{YbTe}$ [8] и два неквазибинарных $\mathrm{Ge}_{0,79} \mathrm{Yb}_{0,21}-\mathrm{Te}$ [9] и $\left[\mathrm{Yb}_{3} \mathrm{Ge}_{5}\right]_{0,45}[\mathrm{GeTe}]_{0,55}-\left[\mathrm{Yb}_{3} \mathrm{Ge}_{7} \mathrm{Te}_{2}\right]_{0,85}[6 \mathrm{YbTe}]_{0,15}$ [10].

Разрез $\mathrm{YbTe}-\mathrm{Yb}_{3} \mathrm{Ge}_{5}$ исследован методами физико-химического анализа: дифференциально-термическим (ДТА), высокотемпературным дифференциально-термическим (ВДТА), рентгенофазовым (РФА), микроструктурным (МСА), а также измерением плотности и микротвердости.

ДТА проводили на пирометре НТР-75 в откачанных до 0,1333 Па кварцевых ампулах: эталоном служил $\mathrm{Al}_{2} \mathrm{O}_{3}$. Скорость нагрева достигала $9-10^{\circ} \mathrm{C} /$ мин.

ВДТА проводили на термоанализаторе марки ВДТА-8М ( $\left.\mathrm{T}_{\text {пл }} \geq 1500-2000 \mathrm{~K}\right)$ по аналогичной методике. Опыты проводили в среде гелия марки ВЧ. Чистота газа 99,999. Образцы для ВДТА готовили в виде прессованных штапиков диаметром $5,7 \cdot 10^{-3}-6 \cdot 10^{-3} \mathrm{M}$ и высотой около $5 \cdot 10^{-3}$ М. В качестве эталонного образца использовали штапик из молибдена. Скорость нагрева и охлаждения составляла $80 \mathrm{~K} / \mathrm{c}$.

РФА порошкообразных образцов проводили на рентгенодифрактометре ДРОН-2 на $\mathrm{CuK}_{\alpha-}$ излучении с Ni-фильтром.

МСА проводили с помощью микроскопа МИМ-8 на предварительно приготовленных шлифах, полированных пастой ГОИ. Травителем служила смесь $1 \mathrm{H} \mathrm{HNO}_{3}+\mathrm{H}_{2} \mathrm{O}_{2}$ в соотношении 2:1, время травления $10-15 \mathrm{c}$.

Микротвердость сплавов измеряли на микротвердомере ПМТ-3 с нагрузкой 0,15 н.

Плотность сплавов определяли пикнометрически, наполнителем служил толуол.

По результатам, полученным методами физико-химического анализа, построена диаграм- 
ма состояния разреза $\mathrm{YbTe}-\mathrm{Yb}_{3} \mathrm{Ge}_{5}$, которая является квазибинарным сечением тройной системы $\mathrm{Ge}-\mathrm{Te}-\mathrm{Yb}$.

При проведении исследования системы использовали германий марки В-4, теллур марки B-3, иттербий марки Итб-1.

Синтез сплавов проводили при температуpe 1450-1700 К и при этой температуре ампула выдерживалась в течении 5-6 ч. Охлаждение проводилось медленно. Сплавы системы синтезировали взаимодействием стехиометрической смеси $\mathrm{Yb}, \mathrm{Ge}$, Te с применением вибрационного перемешивания, причем исходные компоненты (Ge, Те) дополнительно очищались зонной плавкой и вакуумной сублимацией.

После синтеза в ампуле не наблюдалось никаких следов непрореагировавших элементов. Полученные слитки для гомогенизации отжигались в течении 200 ч при 800 К. Однородность синтезированных слитков проверялась методами микростурктурного анализа. Все полученные сплавы системы устойчивы по отношению к воде.

После синтеза и отжига сплавы плотные, компактные, с большим содержанием германия серого цвета с металлическим блеском. Сплавы же, богатые иттербием, пористые и неустойчивые. Минеральные кислоты разлагают их на воздухе.

В таблице приведены составы, результаты ДТА и измерения микротвердости сплавов системы $4 \mathrm{YbTe}-\mathrm{Yb}_{3} \mathrm{Ge}_{5}$.

и измерения микр сти сплавов разреза $4 \mathrm{YbTe}-\mathrm{Yb}_{3} \mathrm{Ge}_{5}$

Table. The composition and results of DTA and microhardness of alloys for section $4 \mathbf{Y b T e}-\mathbf{Y b}_{3} \mathbf{G e}_{\mathbf{5}}$

\begin{tabular}{|c|c|c|c|c|c|}
\hline \multirow[t]{2}{*}{ № } & \multicolumn{2}{|c|}{ Состав, мол.\% } & \multirow{2}{*}{$\begin{array}{c}\text { Термические } \\
\text { эффекты нагре- } \\
\text { вания, К } \\
\end{array}$} & \multicolumn{2}{|c|}{$\begin{array}{c}\text { Микротвердость, } \\
\kappa \Gamma / \mathrm{MM}^{2}\end{array}$} \\
\hline & YbTe & $\mathrm{Yb}_{3} \mathrm{Ge}_{5}$ & & YbTe & $\mathrm{Yb}_{3} \mathrm{Ge}_{5}$ \\
\hline 1 & 100 & - & 2005 & 190 & \\
\hline 2 & 90 & 10 & $915,1025,1600$, & 190 & 685 \\
\hline 3 & 95 & 15 & $915,1025,1160$ & 190 & 685 \\
\hline 4 & 80 & 20 & $915,1025,1180$ & 190 & 685 \\
\hline 5 & 70 & 30 & 915,1025 & 190 & 685 \\
\hline 6 & 60 & 40 & 915,1025 & 190 & 685 \\
\hline 7 & 50 & 50 & 915,1025 & 190 & 685 \\
\hline 8 & 40 & 60 & 915,1025 & 190 & 685 \\
\hline 9 & 30 & 70 & 915,1025 & 190 & 685 \\
\hline 10 & 20 & 80 & 915,1025 & 190 & 685 \\
\hline 11 & 15 & 85 & 915 & \multicolumn{2}{|c|}{ эвтектика } \\
\hline 12 & 10 & 90 & 910,1100 & - & 685 \\
\hline 13 & 5 & 95 & 910,1250 & - & 685 \\
\hline 14 & 0 & 100 & 1355 & - & 685 \\
\hline
\end{tabular}

ДТА показывает, что на термограммах сплавов системы имеют по два эффекта. Полученные эффекты эндотермически обратимые.

В интервале концентраций 15 мол.\%-80 мол.\% $\mathrm{Yb}_{3} \mathrm{Ge}_{5}$ химическое взаимодействие между $\mathrm{YbTe}$ и $\mathrm{Yb}_{3} \mathrm{Ge}_{5}$ ограничено, что обусловлено большой областью расслаивания.

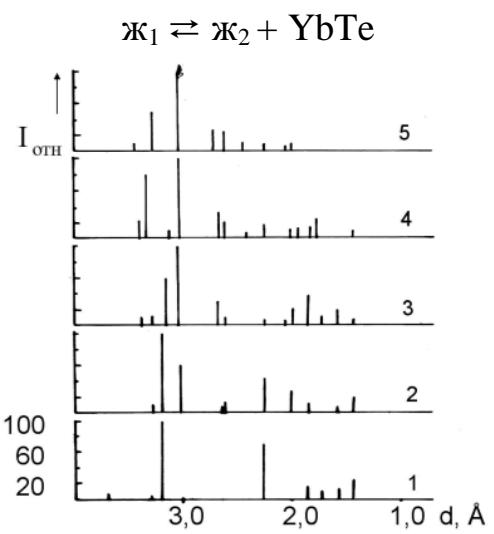

Рис. 1. Штрихдиаграмма сплавов разреза $4 \mathrm{YbTe}-\mathrm{Yb}_{3} \mathrm{Ge}_{5}$ : 1 - YbTe, 2 - 80 мол.\% YbTe, 3 - 50 мол.\% YbTe, 4- 20 мол.\% YbTe, $5-\mathrm{Yb}_{3} \mathrm{Ge}_{5}$

Fig. 1. Stick-diagramma of alloys of $4 \mathrm{YbTe}-\mathrm{Yb}_{3} \mathrm{Ge}_{5}$ section: 1- YbTe, $2-80 \mathrm{~mol} . \%$ of YbTe, $3-50 \mathrm{~mol} . \%$ of YbTe, 4- $20 \mathrm{~mol} \%$ of $\mathrm{YbTe}, 5-\mathrm{Yb}_{3} \mathrm{Ge}_{5}$

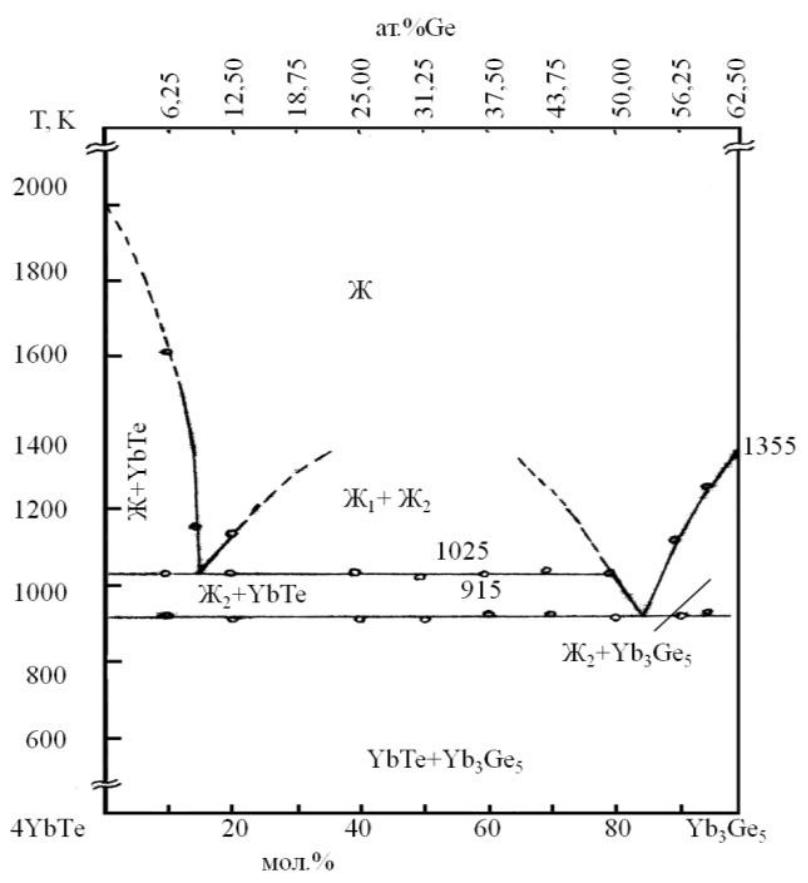

Рис. 2. Диаграмма состояния разреза $4 \mathrm{YbTe}-\mathrm{Yb}_{3} \mathrm{Ge}_{5}$

Fig. 2. State diagram of $4 \mathrm{YbTe}-\mathrm{Yb}_{3} \mathrm{Ge}_{5}$ section

Микроструктура отожженных сплавов показывает, что они двухфазные. Значение микротвердости темной фазы 190 кг/мм² (YbTe), светлой $685 \kappa г / \mathrm{Mм}^{2}\left(\mathrm{Yb}_{3} \mathrm{Ge}_{5}\right)$. В сплавах, содержащих от 15 мол.\% до 80 мол.\% $\mathrm{Yb}_{3} \mathrm{Ge}_{5}$, обнаружено два 
слоя: темный $\mathrm{YbTe}$ и светлый $\mathrm{Yb}_{3} \mathrm{Ge}_{5}$, что характерно для сплавов из области расслаивания.

Для подтверждения данных ДТА, микроструктурного анализа, а также измерения микротвердости был проведен рентгенофазовый анализ. Путем сопоставления штрихдиаграмм установлено, что сплавы системы содержат лишь линии исходных компонентов (рис. 1).

Учитывая результаты ДТА, МСА, РФА и измерения микротвердости сплавов, построена диаграмма состояния разреза $4 \mathrm{YbTe}-\mathrm{Yb}_{3} \mathrm{Ge}_{5}$ (рис. 2).

Как следует из данных, приведенных на рис. 2, в системе химическое взаимодействие меж-

\section{ЛИТЕРАТ УРА}

1. Коржуев М.А. Электросопротивление и термо э.д.с. теллурида германия. Электронная техника. Сер. 6. Материаль. 1983. № 6 (179). С. 33-39.

2. Еременко В.Н., Мелишевич К.А., Буянов Ю.И. Диаграмма состояния системы иттербий-германий. ДАН УССР. Серия А. 1983. № 3. С. 84-89.

3. Ярембаш Е.И., Елисеев А.А. Халькогениды редкоземельных элементов. М.: Наука. 1975. С. 258.

4. Диаграммы состояния двойных металлических систем. Справочник. М.: Машиностроение. 2001. Т. 3. С. 383-384.

5. Мухтарова 3.М., Курбанов Т.Х., Алиев О.М. Система GeTe-YbTe. Журн. неорган. химии. 1985. Т. 30. № 5. C. $1332-1334$.

6. Мухтарова 3.М., Бахтиярлы И.Б., Аждарова Д.С. Фазовое равновесие в системе $\mathrm{GeTe}-\mathrm{Yb}_{3} \mathrm{Ge}_{5}$. Азерб. хим. журн. 2007. № 4. С.155-157.

7. Бахтиярлы И.Б., Мухтарова 3.М., Аждарова Д.С., Мамедов Ф.М. Ликвидус тройной системы $\mathrm{Ge}-\mathrm{Te}-\mathrm{Yb}$. XVIII Мендел. съезд по общей и прикладной химии. Москва. 2007. Т.1. С. 120.

8. Бахтиярлы И.Б., Мухтарова 3.М., Аждарова Д.С., Максудова Т.Ф. О химическом взаимодействии в системах $\mathrm{Yb}_{3} \mathrm{Ge}_{7} \mathrm{Te}_{2}-\mathrm{Ge}, \mathrm{Yb}_{3} \mathrm{Ge}_{7} \mathrm{Te}_{2}-\mathrm{YbTe}$. Хим. проблеMbl. 2007. № 1. C. 92-94.

9. Мухтарова 3.М., Бахтиярлы И.Б., Аждарова Д.С. Политермическое сечение $\mathrm{Ge}_{0,79} \mathrm{YbO}_{, 21}$-Те тройной системы Ge-Yb-Te. Хим. проблемы. 2007. № 2. С. 383384.

10. Мухтарова 3.М., Бахтиярлы И.Б., Аждарова Д.С. Политермическое сечение [Yb3Ge5]0,45[GeTe]0,55[Yb3Ge7Te2]0,85[6YbTe]0,15. Азерб. хим. журн. 2008. № 2. C. $100-101$. ду исходными компонентами отсутствует, что отражается на диаграмме состояния большой областью расслаивания $\mathrm{YbTe}$ и $\mathrm{Yb}_{3} \mathrm{Ge}_{5}$. В интервале концентрации 15-80 мол.\% $\mathrm{Yb}_{3} \mathrm{Ge}_{5}$ происходит монотектическое превращение, которое соответствует изотермической линии при $1025 \mathrm{~K}$.

Таким образом, установлено, что разрез $4 \mathrm{YbTe}-\mathrm{Yb}_{3} \mathrm{Ge}_{5}$ является квазибинарным сечением тройной системы $\mathrm{Ge}-\mathrm{Te}-\mathrm{Yb}$ и диаграмма его относится к эвтектическому типу с монотектикой.

Эвтектика системы $4 \mathrm{YbTe}-\mathrm{Yb}_{3} \mathrm{Ge}_{5}$ отвечает составу 85 мол. $\% \mathrm{Yb}_{3} \mathrm{Ge}_{5}$ и температуре $915 \mathrm{~K}$.

\section{REFERENCES}

1. Korjuyev M.A. The electrical resistance and thermal emf of germanium telluride. Electronic equipment. Ser. 6. Materialy. 1983. N 6. (179). P. 33-39 (in Russian).

2. Yeremenko V.N., Melishevich K.A., Buyanov Yu.I. Phase diagram of ytterbium-germanium system. DAN USSR. Seriya A. 1983. N 3. P. 84-89 (in Russian).

3. Yarembash E.I., Eliseyev A.A. Chalcogenides of rare earth elements. M.: Nauka. 1975. P. 258 (in Russian).

4. The state diagrams of binary metallic systems. Directory. M.: Mashinostroenie. 2001. V. 3. P. 383-384 (in Russian).

5. Mukhtarova Z.M., Kurbanov T.Kh., Aliyev O.M. GeTeYbTe system. Zurn. Neirg. Khimii. 1985. V. 30. N 5. P. 1332-1334 (in Russian).

6. Mukhtarova Z.M., Bakhtiyarly I.B., Azhdarova D.S. Phase equilibria in the system $\mathrm{GeTe}-\mathrm{Yb}_{3} \mathrm{Ge}_{5}$. Azerbaijan Khim. Zhurn. 2007. N 4. P. 155-157 (in Russian).

7. Bakhtiyarly I.B., Mukhtarova Z.M., Azhdarova D.S., Mamedov F.M. Liquidus of ternary system Ge-Te-Yb. XVIII Mendel. Congress on General and Applied Chemistry. Moscow. 2007. V. 1. P. 129 (in Russian).

8. Bakhtiyarly I.B., Mukhtarova Z.M., Azhdarova D.S., Maksudova T.F. On the chemical interaction in $\mathrm{Yb}_{3} \mathrm{Ge}_{7} \mathrm{Te}_{2}-\mathrm{Ge}$, $\mathrm{Yb}_{3} \mathrm{Ge}_{7} \mathrm{Te}_{2}-\mathrm{YbTe}$. Systems. Khim. problemy. 2007. N 1 . P. 92-94 (in Russian).

9. Mukhtarova Z.M., Bakhtiyarly I.B., Azhdarova D.S. Polytermic $\mathrm{Ge}_{0.79} \mathrm{Yb}_{0.21}-\mathrm{Te}$-section triple $\mathrm{Ge}-\mathrm{Yb}-\mathrm{Te}$ system. Khim. problemy. 2007. N 2. P. 383-384.

10. Mukhtarova Z.M., Bakhtiyarly I.B., Azhdarova D.S. Polytermic-section of $\left[\mathrm{Yb}_{3} \mathrm{Ge}_{5}\right] 0.45[\mathrm{GeTe}] 0.55-$ $\left[\mathrm{Yb}_{3} \mathrm{Ge}_{7} \mathrm{Te}_{2}\right] 0.85[6 \mathrm{YbTe}] 0.15$. Azerbaijan Khim. Zhurn. 2008. N 2. P. $100-101$ (in Russian).

Поступила в редакциюю 27.10.2016

Принята к опубликованию 16.01.2017

Received 27.10.2016

Accepted 16.01.2017 